\title{
Shape Accuracy of a Joint-Dominated Deployable Mast
}

\author{
O.R. Stohlman*and S. Pellegrino ${ }^{\dagger}$ \\ California Institute of Technology, Pasadena, CA 91125
}

\begin{abstract}
This paper presents a study to capture and model friction related changes in the unloaded configuration of deployable masts with articulated joints. A finite model of a representative mast structure is described. This model includes a detailed treatment of the latching mechanism. The parameters of the computational model are based on direct measurements on components of a physical model. The moment-rotation relationship for a complete single bay of the physical model has been measured and the overall behavior is predicted well by the model, however the model predicts the residual rotation at zero moment to be zero and so it is concluded that a more refined model for the latch will need to be developed.
\end{abstract}

\section{Introduction}

Deployable masts with lengths up to 60 meters have been used in space for a variety of applications, including the deployment of the International Space Station's solar panels and to maintain the baseline antenna separation for the Shuttle Radar Topography Mission (SRTM). ${ }^{1}$ Masts composed of long jointed trusses are an enabling technology for telescopes and other sensors requiring very long baselines or focal lengths. An x-ray telescope may need to have its optics held 10 meters away from its detector, requiring accuracy on the order of a millimeter and knowledge of the position to microns. The upcoming NuSTAR mission $^{2}$ has requirements of this order. In the future, applications will demand greater and greater precision and predictability of the deployed mast shape.

Many mast models have been addressed in previous studies, including early work on a folding articulated truss $^{3}$ for a tethered satellite experiment, and a number of models of the Astromast, ${ }^{4}$ which has been used to deploy the Mars Odyssey gamma ray spectrometer boom. The recent ST8 SAILMAST ${ }^{5}$ experiment characterized the performance of a coilable boom, appropriate for gossamer structures.

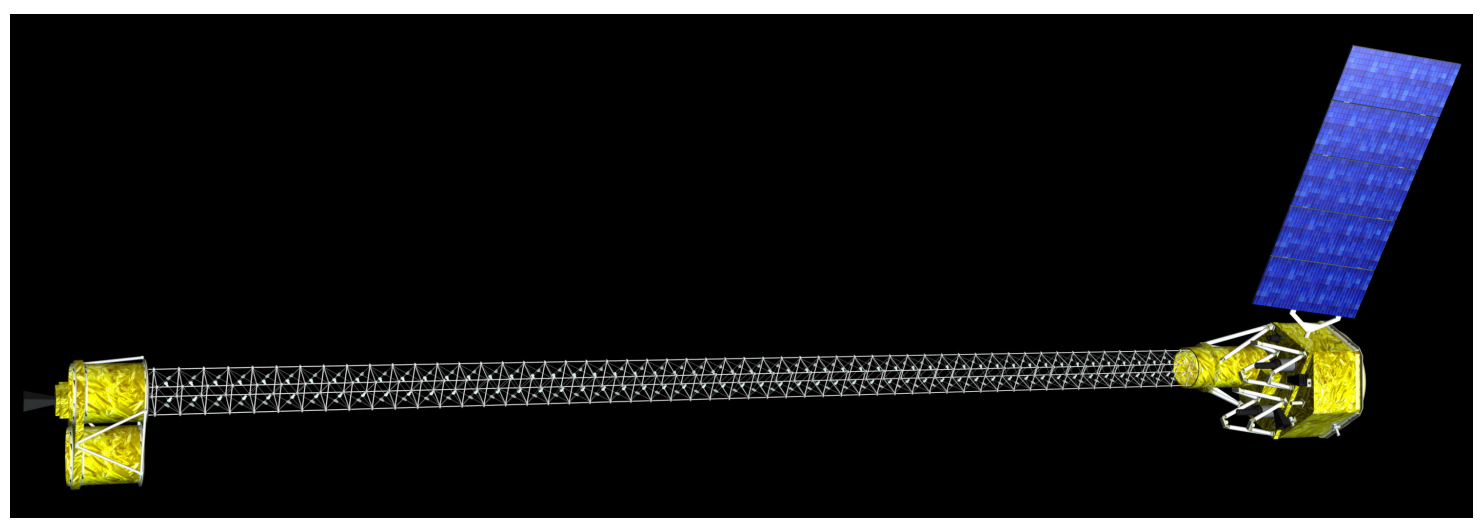

Figure 1. Artist's image of NuSTAR spacecraft, with the optics at left and detectors/spacecraft bus at right. ${ }^{6}$

\footnotetext{
*Graduate student in Aeronautics, Graduate Aerospace Laboratories, 1200 East California Boulevard, Mail Code 205-45, Member AIAA. e-mail: olive@caltech.edu

$\dagger$ Joyce and Kent Kresa Professor of Aeronautics and Professor of Civil Engineering, Graduate Aerospace Laboratories, 1200 E. California Blvd. MC 301-46. AIAA Fellow. sergiop@caltech.edu
} 
As requirements become more stringent, previously unmodeled mechanical details of jointed structures become relevant. Friction in the joints, small errors in manufacturing, thermal expansion coefficient mismatches, and spontaneous dynamic events in the joints all conspire to make control and prediction difficult below the millimeter level. The aim of this research is to characterize, bound, and model the sources of position errors in deployable masts.

Unpredictability in a structure might be grouped into four categories. The first of these, deployment precision, is a situation where misprediction of the deployment process puts the end of a mast in an unanticipated location. The latter three could be grouped under what Lake et $\mathrm{al}^{7}$ describe as "post-deployment stability," here broken down to: static error, where friction or other effects allow the mast to take a new stable shape after a dynamic event, microdynamic error, where unforeseen microdynamic events cause mast motion, and dynamic error, where (due to, for example, gravity- or vacuum-related changes in the frictional preload situation) fundamental frequencies and damping coefficients of the structure are not identified correctly before use. Some of these effects may interact, subtly or otherwise. For example, it has been suggested by Warren et al. ${ }^{8}$ that appropriate transient disturbances to a structure can encourage a series of microdynamic stick-slip events that return a structure to a reliable position. Warren et al. demonstrated a meter-long jointed structure that relaxed via microlurches to a position of sub-micron repeatability.

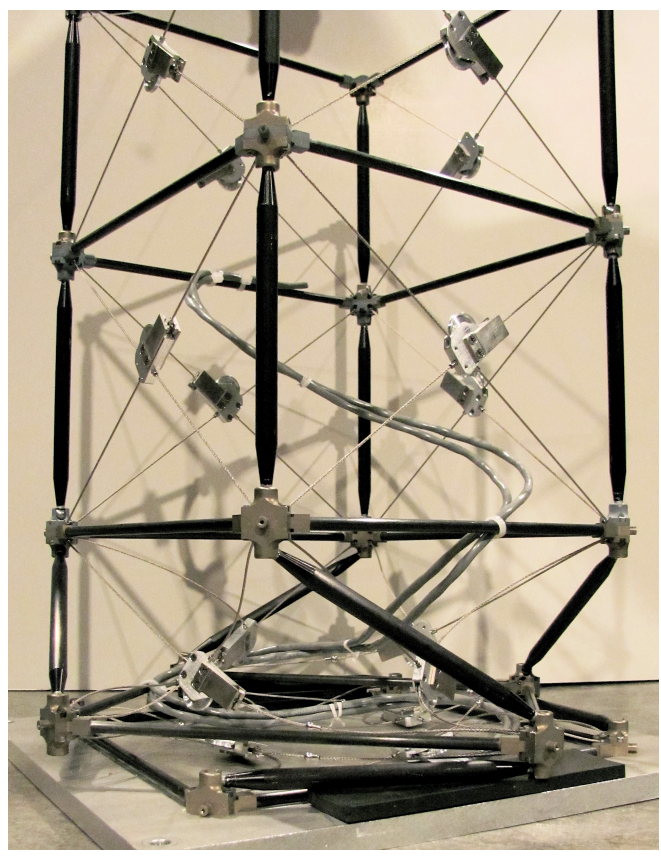

Figure 2. Detail of folded section of ADAM mast.

A wide range of geometries and deployment strategies exist for foldable mast structures. Alliant Techsystems (ATK) produces a modular extensible mast, called ADAM. ${ }^{9}$ Figure 2 shows the transition from stowed to deployed. At the bottom, the stowed bays lie flat. Next, a single bay is shown in an intermediate, partially folded configuration. At the top, the fully deployed bays are shown. Rigid square frames lie at the interface between adjacent bays and are connected to the longerons by ball joints. A simultaneous rotation of the longerons in one bay occurs when the bay is stowed. This modular design allows the ADAM mast to be deployed one bay at a time. Key characteristics of an ADAM mast are the presence of ball joints, reversibly locking elements, and preloaded members.

The ADAM mast is deployed/folded with the aid of a canister structure powered by an electric motor. This motor rotates each bay's top face relative to its bottom face to deploy it into a rectangular prism configuration by forcing the bay's corners along their deployment path.

This paper focuses on masts with the general characteristics of the ADAM family. This particular type of structure was selected because it is the structure of choice for telescope systems. A physical model of this structure was made available for the present study. An ATK technical brochure ${ }^{10}$ quotes a length accuracy and repeatability of $< \pm 1.3 \mathrm{~mm}$ for a mast of $60 \mathrm{~m}$ in length, and $< \pm 0.02^{\circ}$ of rotational misalignment over 
this length. A goal of this research is to capture and model the sources of these errors. In general, friction and gravity effects during ground testing and are two primary causes of static position errors: ${ }^{11}$ in this paper we will focus on friction.

This paper presents a computational model for the position error due to joint friction, and particularly friction's contribution to static position errors. First, a finite model model of a representative mast structure is described. This model includes a detailed treatment of the latching mechanism, based on a user-defined subroutine. The parameters of the computational model are based on direct measurements on components of the physical model. Next, a validation experiment on a complete single bay of the physical model is presented for comparison with the predictions from the computer model. Finally, an example of the predictive potential of the model for a 40-bay mast subject to flexure and torsion is presented.

\section{Modeling of ADAM mast bay}

\section{A. Bay geometry}
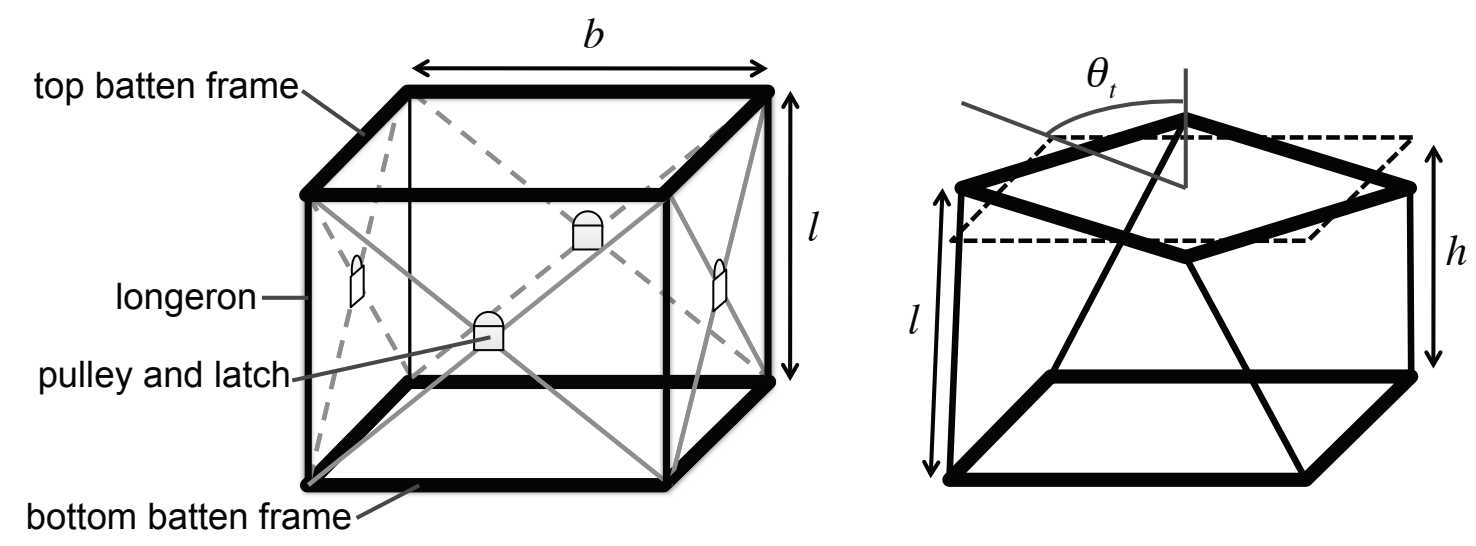

Figure 3. Variables describing the geometry of the system.

Ideally, the deformation of the bay from the folded to the fully deployed configuration should be rotationally symmetric and consist of the top square frame rotating about the central axis of the bay while moving upwards. The relationship between height $h$ and rotation angle $\theta_{t}$ is

$$
h^{2}=b^{2} \cos \theta_{t}-\left(b^{2}-l^{2}\right)
$$

\section{B. Finite-element model}

The finite element analysis software Abaqus ${ }^{12}$ has been used to build a computational model of this deployable mast. The model combines standard Abaqus elements and solution methods with some approaches that are particular to this problem. An analysis of a bay in motion involves large displacements but small strains, and the strain in the stiffer truss members is much smaller than the strain in the cables.

\section{Members and joints}

The longeron and batten members are modeled with two-node cubic beam elements (Abaqus element type B33). This means that they can support bending moments. The material model for these members is linearly elastic and hence appropriate for small strains.

The joints of the structure, which maintain a rigid square shape for the batten frames and support the longeron ball end joints, are modeled as rigid bodies. In reality, these corner pieces are made of metal, and so the material deformation of these joints is considered negligible. ADAM bays typically feature ball joints at the interface between the longeron ends and the corner joints. These are modeled as ball joints with Coulomb friction, i.e. with the conditions that there is no relative motion between the node at the end of the longeron and a coincident node on the joint and that a friction moment exists under most circumstances. In particular, when the joint is in motion and transmits a force $F$, there is a moment opposing the direction of 
motion with the magnitude $|M|=\mu B|F|$, where $B$ is the longeron ball end radius. When the joint is not in motion, the reaction moment must satisfy $|M| \leq \mu B|F|$. The same value of $\mu=0.5$ is assumed for the static and kinetic friction coefficients, and $B=4 \mathrm{~mm}$.

\section{User elements for a system of cables and pulleys}

Because of the complex force-displacement relationship produced by the pulley and latch system on each side of the ADAM bay, these parts must be formulated with special user elements.
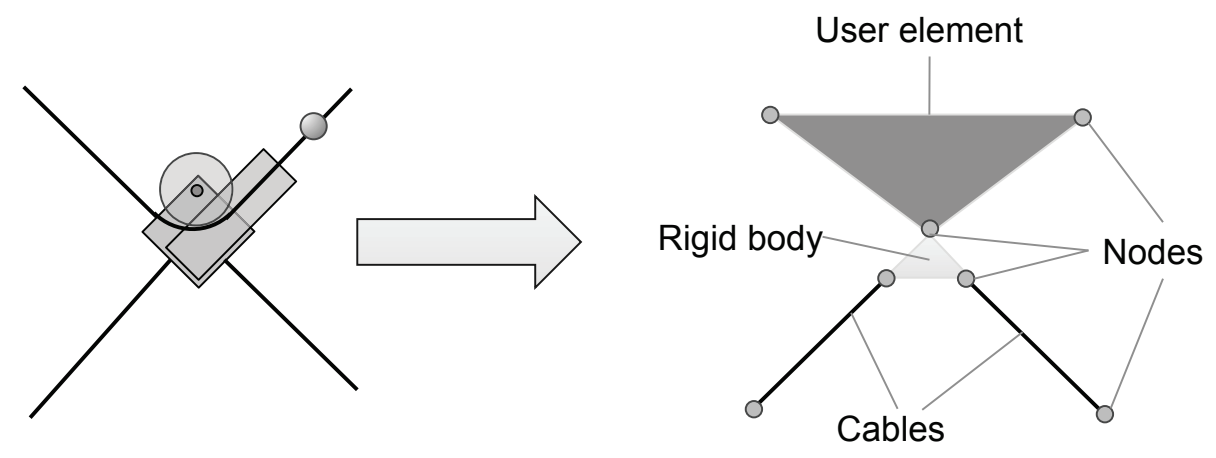

Figure 4. FEA abstraction of the cable assembly on a bay side. The user element is a self-contained subroutine that calculates the reaction forces and stiffness of the pulley, latch, and continuous cable.

A cable that passes over any number of pulleys, latches, or other devices can be described by dividing the cable into straight segments. The distribution of the cable's length between $n$ sections of cable and the cable tension in each section can then be found by solving a system of nonlinear equations.

Consider a cable divided into $n$ sections by $n+1$ anchor points at known locations $\mathbf{x}_{\mathbf{1}} \ldots \mathbf{x}_{\mathbf{n}+\mathbf{1}}$. There is a known constitutive relationship for each section of cable $T_{i}=T_{i}\left(L_{i}, L_{1}^{0}, \ldots, L_{n}^{0}\right)$, where $T_{i}$ is the tension in cable section $i, L_{i}$ is the current (stretched) length of the cable in section $i$ (i.e. $L_{i}=\left|\mathbf{x}_{i+1}-\mathbf{x}_{i}\right|$ ), and $L_{1}^{0}, \ldots, L_{n}^{0}$ are the unstretched lengths of all the sections of cable. As the cable is redistributed over the many pulleys or other elements, the values of $L_{1}^{0}, \ldots, L_{0 n}$ reflect that redistribution and the difference between redistribution of cable and stretching of cable. The total sum of the cable initial lengths will be constant, and equal to $L^{0}$. Finally, each of the $n-1$ pulley or latch elements will have some equation that relates the tension on one side to the tension on the other as a function of the distribution of cable (i.e. an equation of the form $\left.\Delta T_{i}=T_{i+1}-T_{i}=\Delta T_{i}\left(L_{1}^{0}, \ldots, L_{n}^{0}\right)\right)$.

In summary, for $n$ cable sections there are $2 n$ unknowns: $n$ tensions and $n$ unstreched length values. There are also $2 n$ equations: $n$ constitutive relationships, 1 length constraint, and $n-1 \Delta T_{i}$ relationships across the pulley or latch elements.

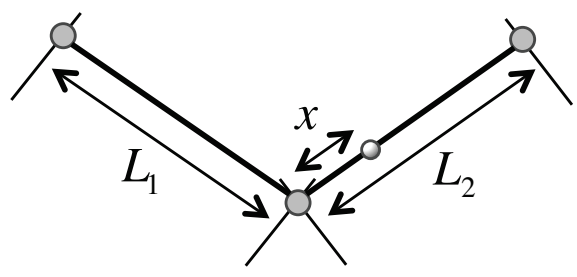

Figure 5. Variables for two-section cable.

The particular design of the ADAM mast requires a cable going over a single pulley-and-latch unit. The following set of equations were used in the user element: 


$$
\begin{gathered}
T_{1}= \begin{cases}0 & L_{1} \leq L_{1}^{0} \\
E A \frac{L_{1}-L_{1}^{0}}{L_{1}^{0}} & L_{1}>L_{1}^{0}\end{cases} \\
T_{2}= \begin{cases}0 & L_{2} \leq L_{2}^{0} \\
E A \frac{L_{2}-L_{2}^{0}}{L_{2}^{0}} & L_{2}>L_{2}^{0}\end{cases} \\
T_{2}-T_{1}=\Delta T(x), \quad x=L_{2}^{0}=L^{0}-L_{1}^{0}
\end{gathered}
$$

Here the cable stiffness $E A$, total unstretched length $L_{0}$, and bead attachment position on the cable $L_{b}$ are part of the element properties. The details of the function $\Delta T(x)$ are discussed in Section III, but when the bead is entirely out of the latch, $\Delta T=0$, and if the bead were to be rigidly fixed in the latch, the equation of $\Delta T$ would be replaced by $L_{1}^{0}=L_{b}$. This model does not account for the size of the pulley, nonlinear stiffness of the cables, or bending stiffness in the cable. The solution of these four equations produces the cable tensions and therefore the reaction forces at the three element nodes.

\section{Boundary conditions and initial step}

In general, it will be assumed that the four corner joints at the base of the mast are rigidly constrained from movement or rotation in any direction. The structure is loaded at a control node located in the center of the top batten frame. This control node is rigidly connected to the four corners of the top batten frame.

The mast is initially defined in the fully deployed configuration. During the initial step of the simulation, the structure must come to equilibrium with the initial internal loads from the cable pretensions. The first step after model initialization may appear unstable and require numerical damping to reach the correct prestressed initial condition. This is accomplished using the *STABILIZE option in Abaqus. No stabilization is used after the initial stable state is achieved.

The analysis of the mast is quasi-static and geometrically nonlinear. The first analysis case considered in this paper is the stowing and deployment of a single bay. This is accomplished in the model by rotating the control node, defining the angle $\theta_{t}$ from Figure 3 .

\section{Measurement and modeling of latch behavior}

A simple analytical model of latch behavior can be developed from some basic assumptions about latch geometry and material behavior.

\section{A. Analytical model}

The latch mechanism can be described as two rollers mounted on cantilever beams. The rollers may rotate with friction about their pins. This mechanism grips a bead that is fixed on the cable that runs through the latch.

The assumed geometry of the latch is shown in Figure 7. It consists of a bead, two rollers, and a backstop. The bead is attached to a cable, which runs through the backstop of the latch. The system is assumed to remain symmetric about the horizontal centerline.

Therefore, while the bead is in contact with the rollers, the following relationship holds:

$$
x^{2}+(h+\delta+r)^{2}=(r+R)^{2}
$$

It is also convenient to introduce the angle between the centerline and the line through the center of the bead and the center of a roller, $\theta$, and note that

$$
\begin{gathered}
\cos \theta=\frac{x}{r+R} \\
\sin \theta=\frac{h+\delta+r}{r+R}
\end{gathered}
$$




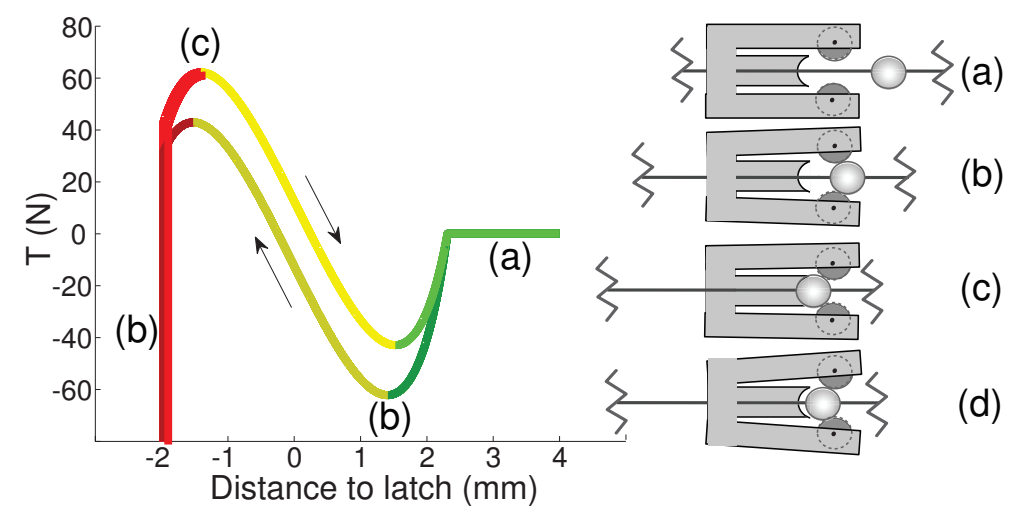

Figure 6. Bead position zones in the latch. Red indicates a completely latched state and green a completely unlatched state, while yellow is intermediate.
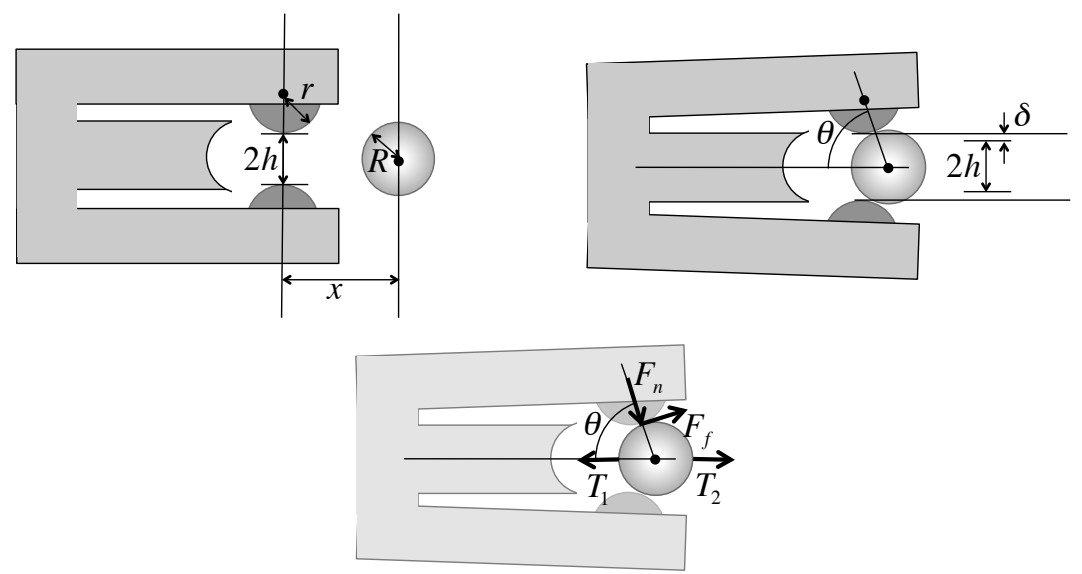

Figure 7. Geometry of the analytical latch model. 
The rollers are assumed to have a linear stiffness, i.e. the force required to open the latch by an amount $2 \delta$ is $F_{\text {pinch }}=k \delta$, where $F_{\text {pinch }}$ is the sum of the vertical components of the forces on the bead.

Now consider the components of the force transmitted between the roller and the bead: the normal component $F_{n}$ and the tangential component $\left|F_{f}\right| \leq\left|\mu F_{n}\right|$. The kinetic and static coefficients of friction are assumed to be equal, so we need only consider the following equations for the two directions of motion:

$$
\Delta T= \begin{cases}-2 F_{n} \cos \theta-\mu F_{n} \sin \theta & d x<0 \\ -2_{n} \cos \theta+\mu F_{n} \sin \theta & d x>0\end{cases}
$$

where $d x$ denotes an infinitesimal change in bead position and $d x>0$ corresponds to the bead moving out of the latch. In the third case, $d x=0$, the bead is subject to static friction, and hence the cable force $\Delta T$ must fall between the two extreme values provided by Eq. 9 .

We can relate $\delta$ with $F_{\text {pinch }}$, and thus $F_{n}$ :

$$
\begin{gathered}
F_{\text {pinch }}=k \delta \\
F_{\text {pinch }}= \begin{cases}F_{n} \sin \theta-\mu F_{n} \cos \theta & d x<0 \\
F_{n} \sin \theta+\mu F_{n} \cos \theta & d x>0\end{cases}
\end{gathered}
$$

Finally, we can calculate the force balance on the bead with respect to $x$ :

$$
\begin{aligned}
\delta & =\sqrt{(r+R)^{2}-x^{2}}-h-r \\
\Delta T & = \begin{cases}-2 k \delta \frac{\cos \theta+\mu \sin \theta}{\sin \theta-\mu \cos \theta} & d x<0 \\
-2 k \delta \frac{\cos \theta-\mu \sin \theta}{\sin \theta+\mu \cos \theta} & d x>0\end{cases} \\
& = \begin{cases}-2 k \delta\left(\frac{x+\mu(h+\delta+r)}{h+\delta+r-\mu x}\right) & d x<0 \\
-2 k \delta\left(\frac{x-\mu(h+\delta+r)}{h+\delta+r+\mu x}\right) & d x>0\end{cases}
\end{aligned}
$$

We can see that there are three geometric parameters in this latch model: the roller radius $r$, the bead radius $R$, and the initial roller separation $h$. There are two parameters that characterize the force relationships: the friction coefficient $\mu$ and the spring stiffness $k$.

There is an additional parameter $x_{\text {backstop }}$ which defines the position at which the bead will come to rest, preventing further motion in the $-x$ direction.

\section{B. Experimental data}

The radius of the bead and the roller separation were measured directly with a micrometer. All other parameters were fitted to experimental data obtained from measurements of an actual latch.

The force-displacement profile of the latch was measured by pulling the bead into and out of the latch by tensioning two opposing cables, as shown in Figure 8. While the latch assembly on the mast will generally have all four cables taut, during this test only two diagonally opposed cables were loaded at a time, leaving the cable on the other side of the latch slack. Because of this, it was not possible to maintain a negative $\Delta T$ during a latching test, nor a positive $\Delta T$ during an unlatching test. An analytical fit was necessitated, in part, by the inability of this testing method to characterize these parts of the latch motion.

Figure 9 shows the raw tension-displacement data obtained from an Instron 5569 material testing machine with a $\pm 1 \mathrm{kN}$ load cell. This measurement was repeated three times in each direction of motion. The position

$x$ of the bead in the latch can be identified by subtracting the stretch of the cables, for example by applying the transformation

$$
x_{i}=d_{i}-\frac{T_{i}}{k_{\text {cable }}}\left(L_{10}+L_{30}\right)-d_{0}
$$

at each data point of cable end-to-end distance and applied tension, $\left(d_{i}, T_{i}\right)$, where $k_{\text {cable }}$ is the cable's previously measured linear stiffness. 


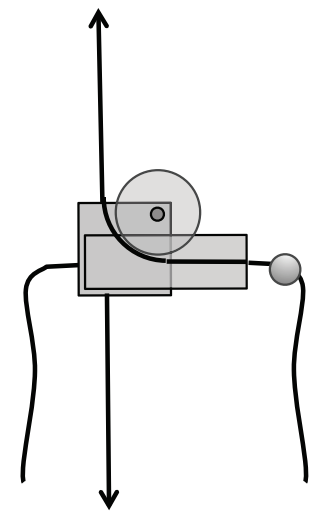

(a) Latching

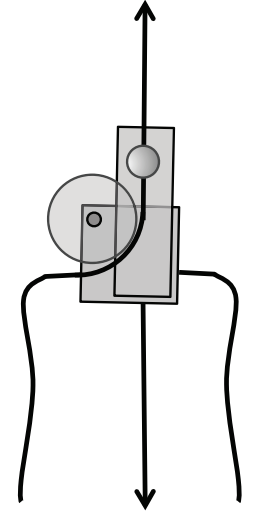

(b) Unlatching

Figure 8. Latch assembly experimental loading. Arrows indicate direction of external loading.

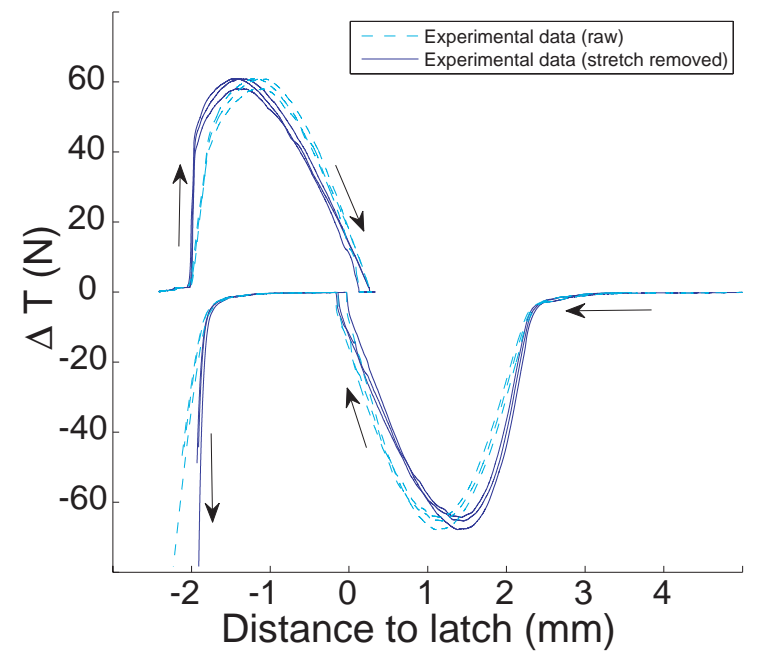

Figure 9. Latch raw data, with and without cable stretch removed. 


\section{Data fit}

The model was fitted to the experimental data using a least-squares method.

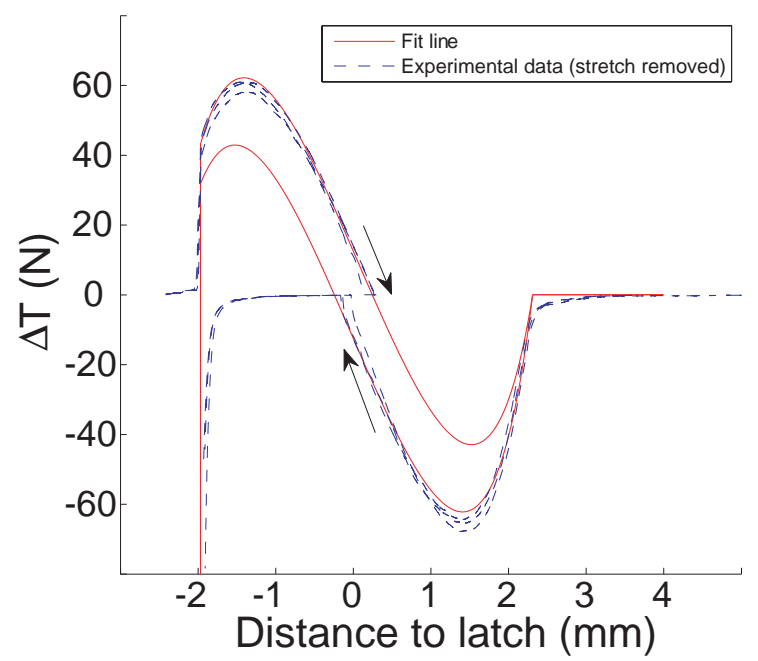

Figure 10. Fit of latch data.

Figure 10 shows the fit data, in red, superimposed on the experimental data. The fit values of the latch variables were $r=1.1 \mathrm{~mm}, x_{\text {backstop }}=2.0 \mathrm{~mm}, k=100 \mathrm{~N} / \mathrm{mm}$, and $\mu=0.07$, with $h=1.5 \mathrm{~mm}$ and $R=2.4$ mm measured before fitting.

Note that fit to the experimental data is generally close, but some smooth transitions have become discontinuous in slope. Also, there is a single curve near the fully latched configuration and so the model assumes no hysteresis in this region.

\section{Measurement of cable pretension}

The level of pretension in the cables on each bay side is an important input to the computational model. Because of the relatively short length of the cables and the particular range of pretensions in the physical model that was tested, commercially available three-point tension meters were unsuitable. Therefore, a vibration method was chosen to measure this parameter in the fully deployed structure.

The method consists in applying a vibrating force to the latch at the center of a bay face and identifying the cable's resonant frequency in out-of-plane motion. There is a monotonic relationship between cable tension and resonant frequency. To quantify this relationship, it was important to get an independent measurement of the cable tensions to correlate with the resonant frequency. Because of the difficulties inherent in measuring the tension of a stranded cable in situ, a mock bay side (shown in Figure 11) with space for inline force sensors (Futek model LCM300, with a capacity of $\pm 250 \mathrm{lbf}$ ) was built.

The mock bay side holds the cable assembly in the same geometry as the actual bay side. The end position of each cable is adjustable so that the cables can be tensioned to different levels. In general, it is difficult to unbalance the tensions in the cables, and only the average tension of the four cables will be considered here.

The latch at the center of the cable assembly was excited by a Labworks Incorporated model ET-132 shaker performing a sine wave sweep from 50 to $100 \mathrm{~Hz}$ over 5 minutes. A force sensor (PBC model 203C01) at the end of the shaker sting measured the applied force, while a Keyence model LK-G87 laser rangefinder tracked the out-of-plane motion of the latch. Measurements were taken at $1000 \mathrm{~Hz}$. Dividing the fast Fourier transform (FFT) of the displacement by the FFT of the applied force, a clear peak can be identified. A set of typical peaks obtained for different cable pretensions is shown in Figure 13.

Plotting the frequency of the resonant peak for the cable assembly at a given cable tension, we find a correlation that is close to linear, as shown in Figure 14. The following relationship between the tension $T$, in $\mathrm{N}$, and the resonant frequency peak $\omega$, in $\mathrm{Hz}$, was obtained by fitting a quadratic polynomial to the 

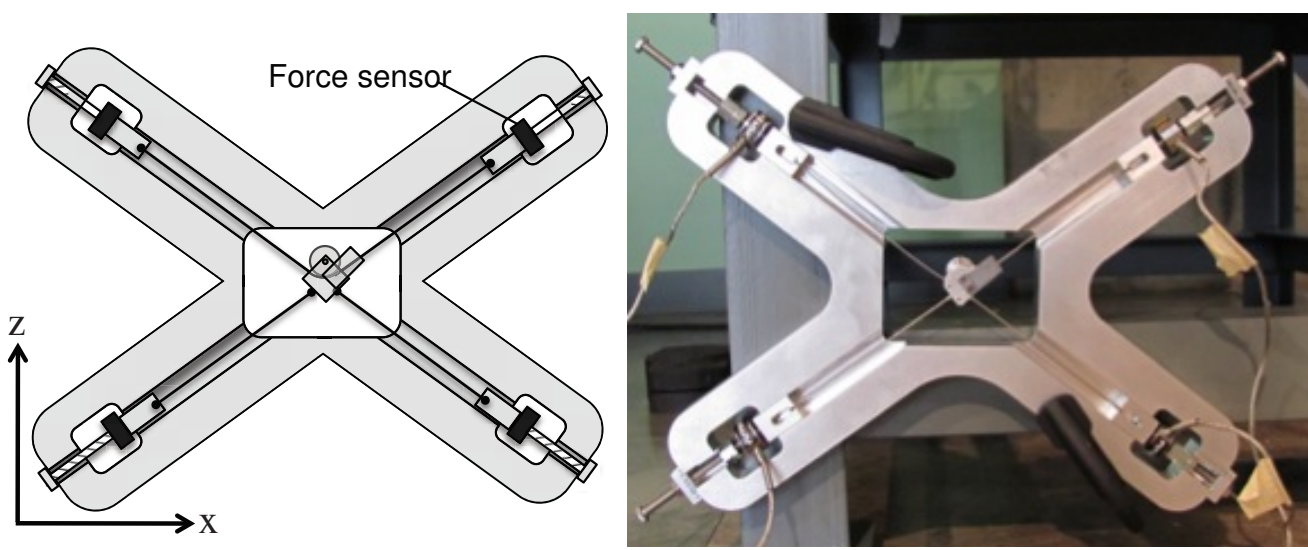

Figure 11. Mock bay side with inline force sensors.

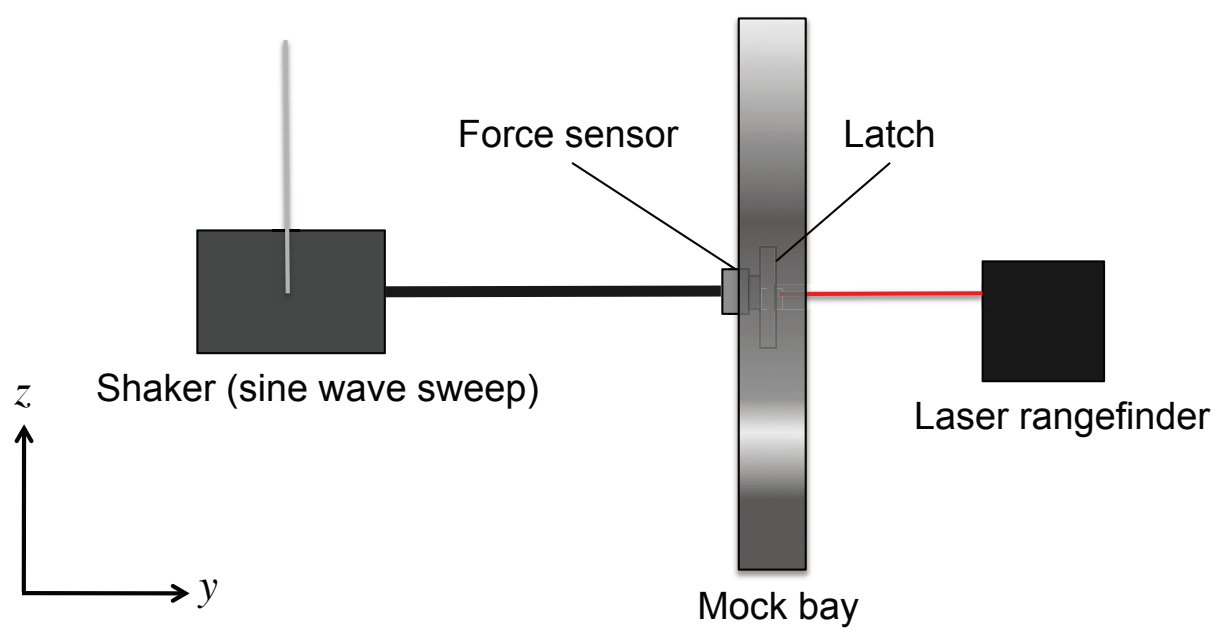

Figure 12. Mock bay (side view) with vibration testing equipment.

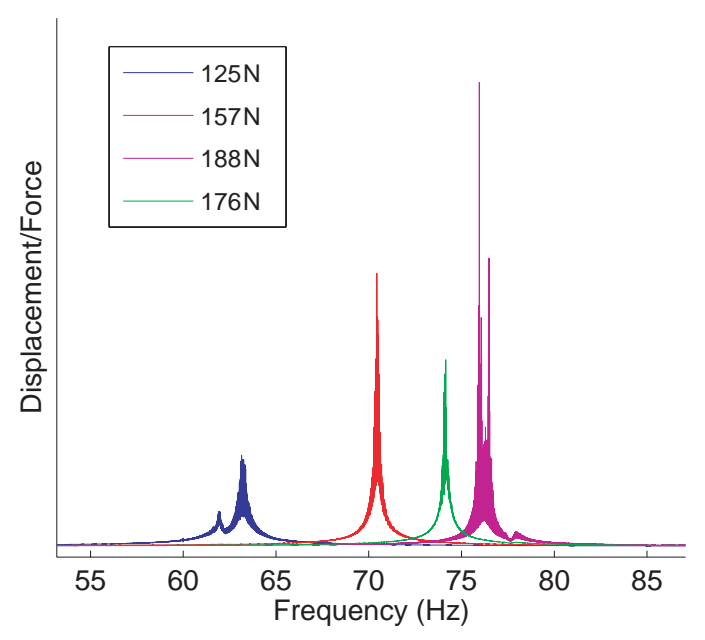

Figure 13. Typical resonance peaks at a variety of cable tensions. 
experimental data:

$$
T=0.012 \omega^{2}-0.58 \omega+17
$$

which fits the 9 data points with a standard deviation of $2 \mathrm{~N}$. This empirical fit was used to estimate the pretension of the cables in the mast.

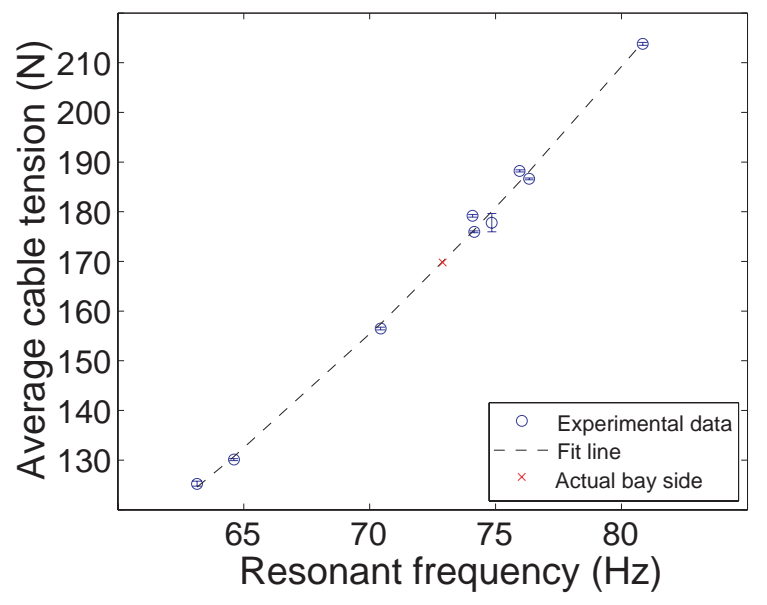

Figure 14. Cable vibration fit data.

\section{Measurement of bay motion}

An experimental characterization of a complete mast bay is necessary as model validation. The test that was chosen is a moment-rotation test. The bay was stowed and deployed by twisting the top batten frame relative to the base. By measuring the applied torsion throughout this motion, a moment-angle relationship for the complete bay unit in torsion can be recorded.

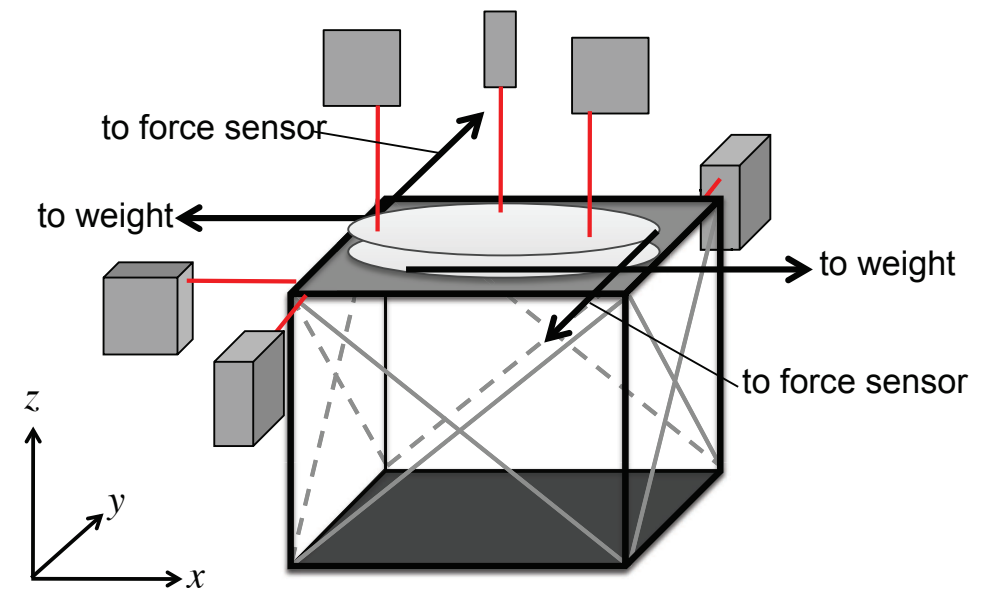

Figure 15. Experimental setup with laser arrangement.

Using six rangefinding lasers, the position and orientation of the top batten frame was tracked at $200 \mathrm{~Hz}$. The arrangement of these lasers is shown in Figure 15. Assuming that the top frame and attached plate are effectively rigid, all six degrees of freedom can be assessed from this data.

The torquing forces are applied through a set of two cables preloaded by weights in one direction (towards the deployed state) and by a manual adjustment mechanism with an inline force sensor in the other. This makes it possible to apply a moment continuously, without a dead band at zero moment. A disadvantage of 
this setup is that it does not provide any constraint keeping the bay aligned or symmetric. As discussed in the following sections, this complicates the modeling problem.

Each experiment was carried out starting in the fully deployed state with the full load of the weights applying a moment of $-35 \mathrm{~N}$ about the $z$ axis. The cables in line with the force sensors were then shortened in steps of a fraction of a millimeter over a period of approximately 15 minutes. After a rotatin of about $3^{\circ}$, three of the four latches had unlatched and the bay no longer provided any resistance to further motion. At this point in the measurement, the motion was reversed and the cables in line with the forces sensors were lengthened. This allowed the weighted cables to apply a deployment torque and the bay returned to the original deployed configuration.

\section{Model validation}

The moment-rotation problem was modeled with two approaches. The first is an idealized model, where the bay is assumed to follow a perfectly symmetric path. This means that, assuming an initially symmetric structure, all four latches experience latching or delatching simultaneously. This is not a realistic description of the experiment, but does represent a baseline scenario of perfect manufacture and deployment. The second model uses the measured $x, y$ translation data as an additional input to the simulation and so is able to capture the asymmetry of the bay's response.

\section{A. Idealized path}

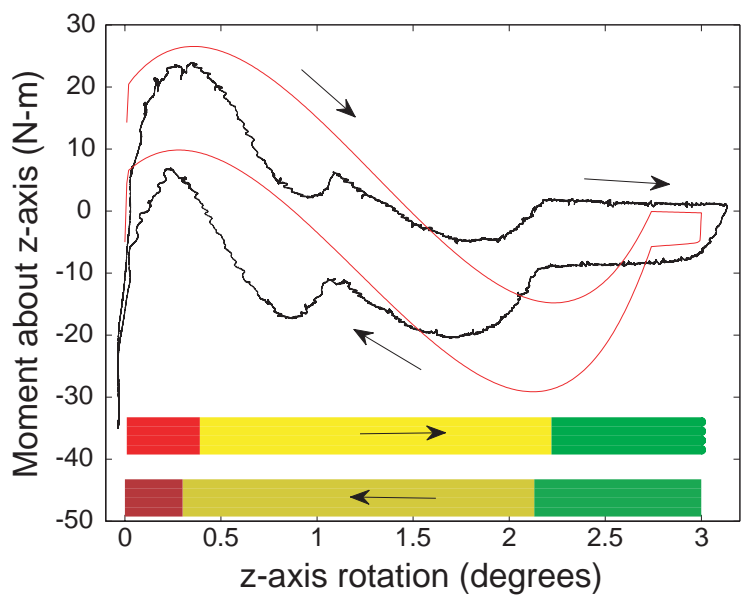

Figure 16. Experimental data (black) compared with computational model of ideal path (red). The colored bars indicate the position of the four cable beads in the four latch assemblies, as described in Figure 6.

Figure 16 shows a typical experimental cycle compared with a symmetric bay cycle simulation. The boundary conditions in this simulation fix the four bottom bay corners in all degrees of freedom. A rigid constraint is enforced between the four top corners of the bay and a control node at the center of the top square. This control node is then stepped through different angles of rotation about the $z$-axis and constrained against motion in the $x$ and $y$ directions. In this simulation no force is applied in any direction.

One reason for the poor agreement between the experimental data and the idealized model is the off-axis motion present in the experiment. Displacements of up to $1 \mathrm{~cm}$ in the $x$ and $y$ directions were recorded, making it impossible for all four latches to be perfectly synchronized.

\section{B. Actual path}

Because the experimentally observed motion does not follow the ideal path, a different approach is needed for a meaningful comparison between the computational model and this experiment. In particular, the model can be run with boundary conditions defined by the measured experimental displacement of the bay top. The measured rotation about the $z$ axis and displacements in the $x$ and $y$ directions were set as rotation and displacement conditions on the control node at the center of the bay top. The results of this model scenario 


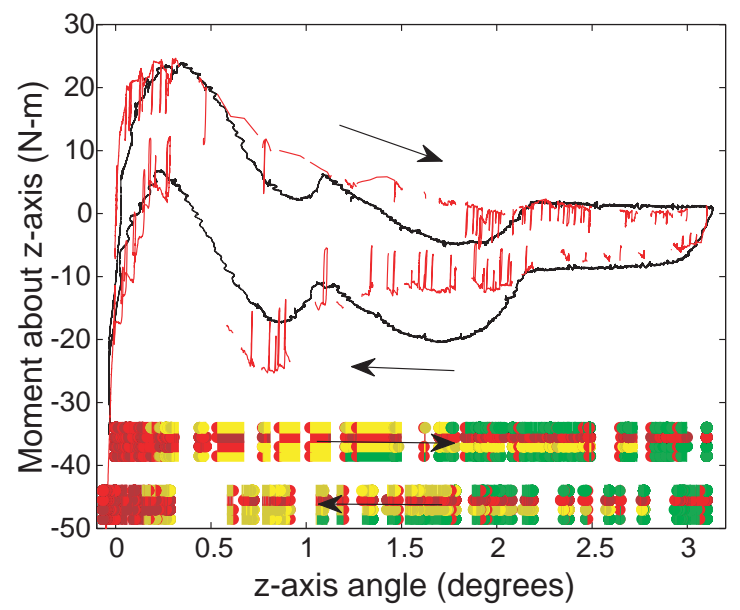

Figure 17. Experimental data (black) compared with FEA model of experimental path (red). The colored bars indicate the position of the four cable beads in the four latch assemblies, as described in Figure 6.

are shown in Figure 17. The colored stripes at the bottom of this graph report the positions of the four beads in their latches throughout the bay motion. These positions are described in Figure 6.

A few features of this figure are worth noting. The record of the latch status on the four sides is consistent with experimental observation. While the latches were not under continuous observation during the experiment, it was consistently noted that one side (although not always the same side) would remain latched after the other three had become unlocked and so the structure had become loose. The double-hump structure of the experimental observation isn't precisely reproduced by the simulation. This characteristic of the moment-angle path is very sensitive to the exact motions of the control node, and inaccuracies in either observing the experimental path or measuring the bay dimensions are reflected.

The spikes in the modeled moment data are related to brief changes in the direction of motion of the bay and the changes in frictional forces caused by these changes. It is not obvious whether this is a spurious result or whether the experimental setup did not capture these spikes. Because there was some compliance in the loading arrangement, this sort of friction sticking during brief periods of backwards motion may have gone undetected. Finally, the gaps in the modeled data are areas where the model failed to converge sufficiently quickly.

\section{Preliminary predictions of mast performance}

One of the applications of a model that includes friction-induced hysteresis is the prediction of residual displacements from transient loading. A 40-bay mast with bays identical to the bay modeled in previous sections is considered here, for a total length of 6.84 meters. During a pointing maneuver in zero gravity, a twisting moment is applied to the mast due to the presence of an offset module at the tip (e.g. telescope optics). The maneuver is assumed to be sufficiently brief that any changes in loading arising from rotation may be neglected; it is also assumed that the inertial forces on the mast are negligible in comparison with the end loading.

The rate of rotational acceleration, $\ddot{\theta}_{x}$, is set at $1.1^{\circ} / \mathrm{s}^{2}$. the off-axis spacecraft module is modeled as a $100 \mathrm{~kg}$ mass offset by $0.50 \mathrm{~m}$ in the $+x$ direction. Therefore, the forces applied at the end of the mast are a torsional moment of $M=6.8 \mathrm{~N} \cdot \mathrm{m}$ about the $z$-axis and a shear force of $F=14 \mathrm{~N}$ in the $x$ direction. To simulate this maneuver, a sequence of $M+F,-M-F$ was applied three times.

Table 1 below shows the position of the control node relative to the base of the mast. This loading pattern produced residual displacements of the mast tip on the order of tenths of $\mu \mathrm{m}$ in the $x$ and $y$ directions and residual changes in angle of the order of arcseconds or tenths of arcseconds of the tip about the $z$ axis. 


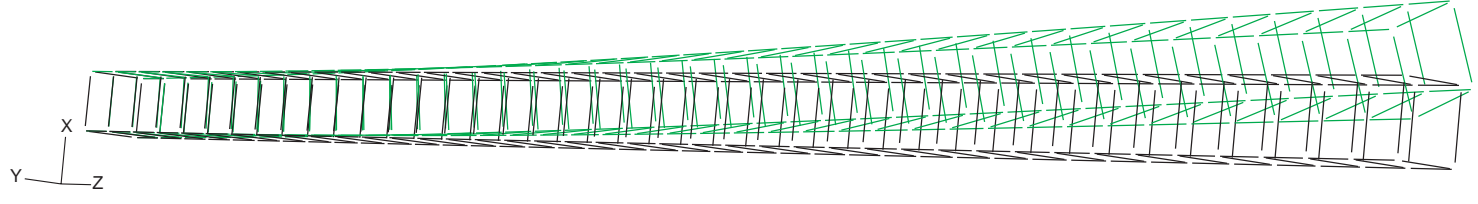

Figure 18. Distorted shape (in green, scaled by a factor of 100) of 40-bay mast under combined bending and torsion load.

\begin{tabular}{c|c|c|c|c|c|c} 
Applied moment & $x(\mu \mathrm{m})$ & $y(\mu \mathrm{m})$ & $z(\mu \mathrm{m})$ & $\theta_{x}(\operatorname{arcsec})$ & $\theta_{y}(\operatorname{arcsec})$ & $\theta_{z}(\operatorname{arcsec})$ \\
\hline \hline 0 & 0.0 & 0.0 & 0.0 & 0.0 & 0.0 & 0.0 \\
\hline$M+F$ & 1740 & -1.0 & -0.3 & 0.6 & 210 & 3030 \\
\hline 0 & 0.8 & -1.0 & 0.0 & 0.0 & 0.02 & 7.9 \\
\hline$-M-F$ & -1.740 & -2.0 & -0.3 & 0.6 & -210 & -3030 \\
\hline 0 & 0.01 & -2.1 & -0.001 & 0.02 & 0.0 & 0.03 \\
\hline$M+F$ & 1.740 & -2.0 & -0.3 & 0.6 & 210 & 3030 \\
\hline 0 & 0.03 & -2.1 & -0.001 & 0.03 & 0.0 & 0.30 \\
\hline$-M-F$ & -1.740 & -2.0 & -0.3 & 0.6 & -210 & -3030 \\
\hline 0 & 0.02 & -2.1 & -0.001 & 0.03 & 0.001 & 0.43 \\
\hline$M+F$ & 1.740 & -2.0 & -0.3 & 0.6 & 210 & 3030 \\
\hline 0 & 0.02 & -2.1 & -0.001 & 0.03 & 0.001 & 0.38 \\
\hline$-M-F$ & -1.740 & -2.0 & -0.3 & 0.6 & -210 & -3030 \\
\hline 0 & 0.02 & -2.1 & -0.001 & 0.03 & 0.001 & 0.38
\end{tabular}

Table 1. Sequence of displacements

\section{Discussion and conclusion}

The strategy of modeling nonlinear joints in detail, presented in this paper, is applicable to many structures. It is especially important in structures whose unpredictability is dominated by the behavior of the joints. The model presented in this paper includes two sources of friction and a nonlinear description of a mast latching system through its full range of motion. This allows residual distortions of the mast shape after transient loading to be investigated. The model's capability to predict the nonlinear moment-rotation relationship of a deployable mast bay through a sequence of stowage and deployment has been demonstrated.

It should be noted that our preliminary predictions in Section VII for the residual displacement due to a load cycle were affected by the latch model's assumption, shown in Figure 10, that there is no residual displacement when $\Delta T$ returns to zero. This is probably the reason for the very small tip rotation that has been predicted. A simple scaling argument, based on 20 of the 40 bays each producing a residual rotation of approximately 0.10 degrees, obtained from the residual rotation at zero moment in the moment-rotation test for the bay, Figures 16 and 17, produces a simple estimate of 2.0 degrees. The reason for considering only half of the bays in this estimate is that in a mast the bays are arranged to fold in alternate directions. This observation suggests that the latch model will need to be refined.

\section{References}

${ }^{1}$ J. W. Umland. SRTM mast damping subsystem design and failure investigation. In 35th Aerospace Mechanisms Symposium, Ames Research Center, 2001.

${ }^{2}$ J. E. Koglin, C.M. Hubert Chen, J. C. Chonko, F. E. Christensen, W. W. Craig, T. R. Decker, C. J. Hailey, F. A. Harrison, C. P. Jensen, K. K. Madsen, M. J. Pivovaroff, M. Stern, D. L. Windt, and E. Ziegler. Hard x-ray optics: From HEFT to NuSTAR. Proc. SPIE, 5488:856-867, 2004.

${ }^{3}$ R. M. Warden. Folding, articulated, square truss. In 21st Aerospace Mechanisms Symposium, L.B. Johnson Space Center, Houston, Texas, April 1987.

${ }^{4}$ L. R. Adams. The x-beam as a deployable boom for the space station. In 22nd Aerospace Mechanisms Symposium, NASA Langley Research Center, Hampton, Virginia, May 1988. 
${ }^{5}$ M. E. McEachen, T. A. Trautt, and D. M. Murphy. The ST8 SAILMAST validation experiment. In 46th AIAA/ASME/ASCE/AHS/ASC Structures, Structural Dynamics, and Materials Conference, Austin, Texas, 2005.

${ }^{6}$ Caltech. Official NuSTAR homepage: http://www.nustar.caltech.edu/, 9th Feb. 2009.

${ }^{7}$ M. S. Lake, L. D. Peterson, M. R. Hachkowski, J. D. Hinkle, and L. R. Hardaway. Research on the problem of highprecision deployment for large-aperture space-based science instruments. In 21st Aerospace Mechanisms Symposium, L.B. Johnson Space Center, Houston, Texas, April 1987.

${ }^{8}$ P. A. Warren, L. D. Peterson, and J. D. Hinkle. Submicron mechanical stability of a prototype deployable space telescope support structure. Journal of Spacecraft and Rockets, 36(5):765-771, 1999.

${ }^{9}$ M. V. Douglas. Module for an articulated stowable and deployable mast. U.S. Patent 5267424, 1993.

${ }^{10}$ ATK Space Systems. Bus structures, towers, and subsystems:

http://www.atk.com/customer_solutions_spacesystems/cs_ss_subsys_bsts.asp, Date 2009.

${ }^{11} \mathrm{~S}$. L. Folkman, E. A. Roswell, and G. D. Ferney. Influence of pinned joints on damping and dynamic behavior of a truss. Journal of Guidance, Control, and Dynamics, 19(6):1398-1403, 1995.

${ }^{12}$ SIMULIA. Abaqus Version 6.8: http://www.simulia.com 